\title{
SOBRE A OCORRÉNCIA DA LUTZOMYIA LONGIPALPIS (LUTZ \& NEIVA, 1912) NA ILHA GRANDE, ESTADO DO RIO DE JANEIRO, BRASIL*
}

\author{
Nelson A. de Araújo Filho** \\ Italo A. Sherlock*** \\ Neide Guitton****
}

\begin{abstract}
Os autores descrevem pela primeira vez a presenca de Lutzomyia longipalpis na Praia Verme/ha, praia localizada na llha Grande, litoral do Estado do Rio de Janeiro e acentuam a importância epidemiológica do achado.
\end{abstract}

Até recentemente era desconhecida a real prevalência da Lutzomyia longipalpis no Estado do Rio de Janeiro, díptero este incriminado como principal vetor de Calazar para o homem e que sempre está presente nas áreas de Calazar do Brasil e de outros países da América do Sul, como assinalam Amaral \& cols. ${ }^{1}$, Biagi \& cols. ${ }^{6}$, Deane ${ }^{7}$, Mangabeira Filho ${ }^{11}$. Martins \& cols. $^{12}$ e Sherlock \& Guitton ${ }^{14}$.

A presença desse flebotomineo no Estado do Rio de Janeiro já tinha sido contudo assinalada, primeiramente por Lutz \& Neiva em 1912, a partir de um exemplar fêmea capturado no município de Mangaratiba; entretanto este achado foi posteriormente posto em dúvida por alguns autores que limitaram sua distribuição geográfica ${ }^{4}, 5,7$, 10. Anos depois, Forattini \& cols. ${ }^{9}$, em 1971, registraram a presença de 19 exemplares da L. longipalpis no bairro do Arado, muni cípio do Salto do Pirapora, Estado de São Paulo.

Em 1974, na eclosão da epidemia da Leishmaniose Tegumentar Americana, em Jacarepaguá, Estado do Rio de Janeiro, foram coletados 5 exemplares da $L$. longipalpis. ${ }^{18}$

No mês de setembro de 1977 um caso autóctone de Calazar humano foi registrado no bairro de Bangú, área suburbana do Estado do Rio de Janeiro. Na procura do vetor alado foram capturados 1.585 flebotomíneos, sendo L. longipalpis a segunda espécie mais coletada, totalizando 684 exemplares $^{13}$.

Em estudos realizados sobre um surto humano de Leishmaniose Tegumentar Americana, durante os anos de 1976/1977, na localidade de Praia Vermelha, Il ha Grande, município de Angra dos Reis, Estado do Rio de Janeiro, investigou-se também a ocorrência dos transmissores dessa doença na área ${ }^{2}, 3$

Nas pesquisas efetuadas durante o período de fevereiro de 1976 a abril de 1977 foram realizadas capturas em domicílios, galinheiros, abrigos naturais, tendo-se, ainda, utilizado animais domésticos e silvestres como iscas. Coletou-se um total de 4.192 flebotomíneos, sendo as espécies prevalentes Lutzomyia intermedia e Lutzomyia migonei. Em 3 galinheiros e no interior de um domicílio, foram coletados 43 exemplares da $L$. longipalpis (Fig. 1), sendo 36 machos e 7 fêmeas, representado a terceira espécie de flebotomíneo mais coletada na área.

* Trabalho do Departamento de Medicina Preventiva da Faculdade de Medicina da Universidade Federal do Rio de Janeiro, realizado com auxílio financeiro do CNPq.

* Docente da Universidade do Amazonas. Mestre em Doenças Infecciosas e Parasitárias pela Universidade Federal do Rio de Janeiro.

* * Pesquisadores da Fundação Oswaldo Cruz - Centro de Pesquisas Gonçalo Muniz, Bahia. 
Apesar de nẫo haver sido relatada a presença de Calazar autóctone naquela área de trabalho e sendo atualmente conhecida apenas a ocorrência de Leishmaniose Tegumentar Americana ${ }^{2}{ }^{3}$ a presença da $L$. longipaldis poderá vir a ter importância em vista da possibilidade do aparecimento de casos da doença visceral.

O encontro da $L$. longipalpis, no Estado de São Paulo, e atualmente nas duas localidades referidas no Estado do Rio de Janeiro, assume elevada importância em termos de saúde pública. Sabe-se que migrações intérnas para o Estado do Rio de Janeiro são uma constante, principalmente de populações procedentes de áreas de Calazar do país. A ocorrência da forma inaparente do Calazar e mesmo de casos clinicamente estabelecidos podem provocar a disseminação da doença no Estado do Rio de Janeiro, pois a presença do vétor alado já poderá manter uma epidemia de conseqüências graves, levando-se em consideração que o nivel imunitário da população do Estado do Rio de Janeiro provavelmente é baixo ou quase nulo para o Calazar.

Um sistema de vigilância sanitária deve ser orientado para o diagnóstico do Calazar, do contrário, o Estado do Rio de Janeiro, poderá registrar mais uma doença de prognóstico grave e muitas vezes fatal no rol das endemias transmitidas por vetores.

\section{SUMMARY}

For the first time it has been detected. Lutzomyia longipalpis at Praia Vermelha, a beach of an offshore island of the Rio de Janeiro State (Ilha Grande), Brazil and attention must be paid to its possible epidemiological importance.

\section{REFERÉNCIAS BIBLIOGRÁFICAS}

1. AMARAL, A.D.F., TORREALBA, J.W., HENRIQUEZ, C.C., KOWALENKO, W. \& BARRIOS, P.A. - Phlebotomus longipalpis (LUTZ \& NEIVA, 1912) probable transmissor de la leishmaniasis visceral en Venezuela. Gaz. Med., Caracas, 70: 389-408, 1961.

2. ARAÚJO FILHO, N.A., WANKE, B, COUTINHO, S.G. \& COURA, J.R. - Surto de leishmaniose tegumentar americana na llha Grande. In Cong. Soc. Bras. Med. Trop., 12!, Belém, 1978.

3. araÚjo FILHO, N.A. - Epidemiologia da leishmaniose tegumentar americana na llha Grande, Rio de Janeiro. Estudos sobre a infecção humana, reservatórios e transmissores. Tese de Mestrado, Pós-graduação em doenças Infecciosas e Parasitárias. Fac. Med. da Universidade Federal do Rio de Janeiro, Rio de Janeiro, 148 p, 1978.

4. BARRETTO, M.P. - Catálogo dos flebotomíneos americanos. Arq. Zool. Est. São Paulo, 5: 177-242, 1974.

5. BARRETO, M.P. - Nova contribuição para o estudo da distribuição geográfica dos flebotomos americanos (Diptera, Psychodidae). Arq. Hig. e Saúde Públ. S. Paulo, 13: 211-226, 1950.

6. BIAGI, A.M. de B., BIAGI, F.F. \& MELINARI, J.L. - Kala-azar en México. Antropofilia y actividade horária de Phlebotomus longipalpis LUTZ \& NEIVA, 1912 (Diptera, Psychodidae). Rev. Inst. Salubr. Enferm. trop., México, 25: 13-9, 1965.

7. DEANE, L.M. - Leishmaniose visceral no Brasil. Estudos sobre reservatórios e transmissores realizados no Estado do Ceará. Serviço Nacional de Divulgação Sanitária, Rio de Janeiro, 162 p, 1956.

8. FUNDAÇÃO OSWALDO CRUZ (FIOCRUZ) - Relatório do grupo de trabalho coordenador da atividade de estudo e controle da Leishmaniose Tegumentar Americana na área de atuação do posto Samuel Libânio (Jacarepaguá, Rio de Janeiro, 25 p.), 1974.

9. FORATTINI, O.P., RABELLO, E.X. \& PATOLLI, D. - Sobre o encontro da Lutzomya longipalpis (LUTZ \& NEIVA, 1912) no Estado de São Paulo, Brasil. Rev. Saúde Públ. S. Paulo, 4: 99-100, 1971 .

10. LIMA, A.M. da COSTA - Flebótomos americanos (Diptera, Psychodidae). 
Mem. Inst. Oswaldo Cruz, 26: 15-69, 1932.

11. MANGABEIRA Fitho, O. - Sobre a sistemática e biologia dos flebótomos do Ceará. Rev. Bras. Malariol., 21: 3-26, 1969.

12. MARTINS, A.V., BRENER, Z., MOURÃO, D.G., LIMA, M.M., SOUZA, M.A. de \& SILVA, J.E. da Calazar autóctone em Minas Gerais. Rev. Bras. Malariol., 8: 554-563, 1956.

13. SABROZA, P.C., SOUZA, M.A. \& MARZOCHI, M.C.A. - Flebotomineos na cidade do Rio de Janeiro. In Cong.
Bras. Med. Trop., 14?, João Pessoa, 1978.

14. SHERLOCK, I.A. \& GUITTON, N. Observações sobre o Calazar em Jaco. bina, Bahia. II. Alguns dados sobre o Phlebotomus longipalpis, o principal transmissor. Rev. Bras. Malariol., 21: 541-548, 1969.

\section{Agradecimentos:}

Os autores agradecem a colaboração da técnica de entomologia Célia Maria Dias Lima do Centro de Pesquisas Gonçalo Muniz - FIOCRUZ, Bahia, pela preparação para taxonomia dos flebotomíneos deste trabalho. 